\title{
Conformal Inflation, Modulated Reheating, and WMAP5
}

\author{
Takeshi Kobayashi1, 田 and Shinji Mukohyama, ${ }^{2,0}$ \\ 1 Department of Physics, School of Science, The University of Tokyo, \\ Hongo 7-3-1, Bunkyo-ku, Tokyo 113-0033, Japan \\ 2 Institute for the Physics and Mathematics of the Universe (IPMU), \\ The University of Tokyo, 5-1-5 Kashiwanoha, Kashiwa, Chiba 277-8582, Japan
}

\begin{abstract}
We investigate density perturbations generated through modulated reheating while inflation is driven by a conformally coupled scalar field. A large running of the spectral index is obtained, which reflects the basic nature of conformal inflation that higher-order time derivatives of the Hubble parameter during inflation are not necessarily small. This feature may allow us to distinguish between conformal inflation models and standard minimally coupled ones. We also investigate how the resulting fluctuations are modified when there is a deviation from an exact conformal coupling between the inflaton and gravity. Finally, we apply our results to the warped brane inflation model and see that observational bounds from the WMAP5 data suggest a blue tilted density perturbation spectrum.
\end{abstract}

\section{INTRODUCTION}

Ever since the idea of cosmic inflation was proposed, inflationary model building has largely focused on slowrolling scalar fields minimally coupled to gravity as candidate inflatons. However, recently it was pointed out in [1] that conformally coupled scalar fields are also capable of accelerating the universe. Since the existence of such conformally coupled fields is rather common in models from string theory, it is of great interest to explore the possibility of conformally coupled fields driving inflation. In this light, we also come up with a new question of how we can distinguish between these "conformal inflation" models and standard minimally coupled ones.

The aim of this paper is to focus on density perturbations and seek distinctive features conformal inflation might have left. A conformally coupled inflaton itself cannot be responsible for generating primordial fluctuations $^{1}$, but instead string theory suggests alternative scenarios such as modulated reheating [3, 4]. Hence, in this paper we consider the case where modulated reheating generates density perturbations while inflation is driven by an almost conformally coupled inflaton. ${ }^{2}$ We study the scale dependence of the generated density perturbations. The general results we obtain are that (i) modulated reheating together with conformal inflation can produce a nearly scale-invariant spectrum, and (ii) the

\footnotetext{
*tkobayashi@utap.phys.s.u-tokyo.ac.jp

shinji.mukohyama@ipmu.jp

${ }^{1}$ From a conformally coupled inflaton, one generally obtains a highly blue tilted spectrum. (This can easily be seen by moving to the Einstein frame and computing the density perturbations [2].) Therefore, unless we consider inflation with an extremely high energy scale, perturbations generated by the inflaton will be suppressed at the CMB scale.

2 One may wonder whether the coupling of the inflaton to Standard Model particles may spoil conformal symmetry. However, we need not require an exact symmetry, instead, the basic factors for conformal inflation are the inflaton's (almost) conformal coupling to gravity together with an appropriate inflaton potential.
}

running of the spectral index $\left|d n_{s} / d \ln k\right|$ turns out to be as large as $\left|n_{s}-1\right|$. The latter result reflects the nonexistence of hierarchy among higher-order time derivatives of the Hubble parameter $d^{n} \ln H^{2} / d t^{n} H^{n}$ during conformal inflation. This is in strong contrast to standard minimal models where higher-order derivatives are suppressed by higher orders of the slow-roll parameters (and their derivatives). Hence this feature offers a chance to obtain a smoking-gun signal for nonminimally coupled inflation models.

We also investigate how the resulting density perturbations are modified when there is a deviation from an exact conformal coupling between the inflaton and gravity. The result will allow us to impose constraints on the inflaton's coupling from observational data.

As a concrete example, our generic results are applied to the well-studied warped brane inflation model [5, 6, 7, 8, 9, 10]. We will see that comparison with the WMAP5 data [11] predicts a blue tilt $\left(n_{s}>1\right)$ for this model.

The paper is organized as follows. First we give a quick review on conformal inflation in Section III. In Section we study the scale dependence of the density perturbations generated through modulated reheating after conformal inflation. Then in Section IV we apply our results to the warped brane inflation model. We conclude in Section $\mathrm{V}$. In the appendix we provide detailed arguments on conformal inflation.

\section{REVIEW OF CONFORMAL INFLATION}

Here we give a brief review of conformal inflation. Consider the action

$S=\int d x^{4} \sqrt{-g}\left[\frac{M_{p}^{2}}{2} \mathcal{R}-\frac{1}{2} g^{\mu \nu} \partial_{\mu} \phi \partial_{\nu} \phi-V(\phi)-\frac{\xi}{2} \mathcal{R} \phi^{2}\right]$

where $\mathcal{R}$ is the scalar curvature and $\xi$ is the nonminimal coupling to gravity. Choosing a flat FRW background,

$$
d s^{2}=-d t^{2}+a(t)^{2} d \mathbf{x}^{2}
$$


the Friedmann equation is

$$
\left(M_{p}^{2}-\xi \phi^{2}\right) H^{2}=\frac{1}{6} \dot{\phi}^{2}+\frac{1}{3} V(\phi)+2 \xi H \phi \dot{\phi},
$$

and the equation of motion of $\phi$ is

$$
\ddot{\phi}+3 H \dot{\phi}+V^{\prime}(\phi)+6 \xi\left(\dot{H}+2 H^{2}\right) \phi=0 .
$$

By introducing

$$
\pi \equiv \dot{\phi}+H \phi
$$

the equations (3) and (44) can be rewritten in the following form:

$$
\begin{gathered}
M_{p}^{2} H^{2}=\frac{1}{3} V+\frac{1}{6} \pi^{2}+\left(\xi-\frac{1}{6}\right)\left(2 H \phi \pi-H^{2} \phi^{2}\right), \\
\dot{\pi}+2 H \pi+V^{\prime}+6\left(\xi-\frac{1}{6}\right)\left(\dot{H}+2 H^{2}\right) \phi=0 .
\end{gathered}
$$

The conformal case $\xi=1 / 6$ was investigated in [1], where inflation was realized while the equations (6) and (7) could be approximated to

$$
\begin{aligned}
& M_{p}^{2} H^{2} \simeq \frac{1}{3} V, \\
& c H \pi \simeq-V^{\prime} .
\end{aligned}
$$

Here, $c$ is a dimensionless constant whose meaning will soon become clear ${ }^{3}$. Let us define three "flatness parameters" as

$$
\begin{aligned}
\epsilon & \equiv \frac{M_{p}^{2}}{2}\left(\frac{V^{\prime}}{V}\right)^{2}, \\
\tilde{\epsilon} & \equiv \frac{\phi V^{\prime}}{2 V}, \\
\eta_{c} & \equiv \eta+\frac{c}{3}\left(\frac{V^{\prime \prime} \phi}{V^{\prime}}+c-2\right),
\end{aligned}
$$

where

$$
\eta \equiv M_{p}^{2} \frac{V^{\prime \prime}}{V} .
$$

Then one can check that the necessary conditions for the approximations (8) and (9) to be valid are

$$
\frac{6}{c^{2}} \epsilon \ll 1, \quad\left|\frac{6}{c^{3}} \epsilon+\frac{1}{c} \tilde{\epsilon}-\frac{3}{c^{2}} \eta_{c}\right| \ll 1 .
$$

The necessary condition for $\left|\dot{H} / H^{2}\right| \ll 1$ can also be derived,

$$
\left|\frac{6}{c^{2}} \epsilon+\tilde{\epsilon}\right| \ll 1
$$

\footnotetext{
${ }^{3}$ Our parameterization of $c$ differs from that of [1] by $c_{\text {ours }}=$ $c_{[1]}+2$.
}

When $|c| \sim \mathcal{O}(1)$, these conditions take the simple form

$$
\epsilon \ll 1, \quad|\tilde{\epsilon}| \ll 1, \quad\left|\eta_{c}\right| \ll 1 .
$$

The proportionality constant $c$ is chosen such that it is the largest constant to minimize $\left|\eta_{c}\right| / c^{2}$. For detailed arguments, see Appendix A 1 .

It is clear from (6) and (86) that $\pi^{2} / V$ is small during inflation. Especially when the potential is flat enough to satisfy $\left|M_{p}^{2} V^{\prime}\right| \ll|c \phi V|$, then

$$
\dot{\phi} \approx-H \phi
$$

which suggests that the inflaton is rapidly rolling towards its origin.

\section{DENSITY PERTURBATIONS FROM MODULATED REHEATING}

We analyze the case where density perturbations are generated through modulated reheating [3, 4] after conformal inflation. The scale dependence of the perturbations will be expressed in terms of the flatness parameters and $(\xi-1 / 6)$.

\section{A. Modulated Reheating Scenario}

To reheat the universe, the inflaton should decay into ordinary Standard Model particles (or particles that eventually transfer their energy into SM particles) after inflation. Here, it may well be that the decay rate of the inflaton field (i.e. couplings between the inflaton and ordinary particles) is determined by VEVs of fields in the theory. If those fields are light during inflation, they will fluctuate and generate spacial fluctuations in the decay rate for the reheating process, which translate into density perturbations in the universe.

Let us take $\chi$ to be the light modulus field whose VEV determines the coupling $\lambda$. Then its fluctuations during inflation manifest themselves as fluctuations in $\lambda$ and the decay rate $\Gamma$, leading to density perturbations

$$
\frac{\delta \rho}{\rho} \propto \frac{\delta \Gamma}{\Gamma} \propto \frac{\delta \lambda}{\lambda} \propto \frac{\delta \chi}{\chi} .
$$

Fields whose mass is smaller than $H$ during inflation typically obtain fluctuations of order $H$. Therefore the tilt of the spectrum in this scenario can be expressed in a form independent of the details of the model,

$$
\begin{aligned}
n_{s}-1 & =\frac{d \ln H^{2}}{d \ln k}=2 \frac{\dot{H}}{H^{2}}\left(1+\frac{\dot{H}}{H^{2}}\right)^{-1} \\
\frac{d n_{s}}{d \ln k} & =2\left(1+\frac{\dot{H}}{H^{2}}\right)^{-3} \frac{1}{H}\left(\frac{\dot{H}}{H^{2}}\right)
\end{aligned}
$$


where the spectral index and its running are estimated at the moment of horizon crossing $k=a H$.

We do not restrict ourselves to specific setups of modulated reheating in this paper. Rather, we focus on the generic features (19), (20) of modulated reheating and study the imprints of conformal inflation on the tilt of the spectrum. (Some candidates for the light modulus field in the case of warped brane inflation are proposed in Section V.)

\section{B. The Spectral Index and its Running}

In this subsection, the observables are expanded in terms of the three flatness parameters $\epsilon, \tilde{\epsilon}$, and $\eta_{c}$. We assume $|c| \sim \mathcal{O}(1)$. From now on, we take account of the inflaton's slight deviation from a conformal coupling $(\xi-1 / 6)$. This procedure allows us to deal with a wide variety of situations, e.g., when there exists additional corrections to the action which ruin the exact conformal coupling, when the frame where the inflaton $\phi$ is conformally coupled to gravity differs from the frame where the light modulus $\chi$ is minimally coupled.

Since the flatness parameters only describe the form of the potential, let us introduce an additional dimensionless parameter $\alpha$ as follows,

$$
-V^{\prime}=c H \pi+\frac{V}{M_{p}} \alpha .
$$

First we make use of this parameter to evaluate quantities, and then later on we will estimate the value of $\alpha$ itself. For convenience, we further define

$$
e \equiv \frac{M_{p} V^{\prime}}{\sqrt{2} V}, \quad \kappa \equiv \frac{\phi}{M_{p}}, \quad \sigma \equiv \frac{\pi^{2}}{V}
$$

Note that $\epsilon=e^{2}$ and $\tilde{\epsilon}=e \kappa / \sqrt{2}$. Then (21) can be rewritten as

$$
\sigma=\frac{V}{c^{2} M_{p}^{2} H^{2}}(\alpha+\sqrt{2} e)^{2}
$$

Also, from (6) and (21) one can derive

$$
\frac{3 M_{p}^{2} H^{2}}{V}=\frac{1+\frac{1}{2} \sigma-\frac{1}{c}(6 \xi-1) \kappa(\alpha+\sqrt{2} e)}{1+\frac{1}{6}(6 \xi-1) \kappa^{2}} .
$$

(23) and (24) give a quadratic equation on $\sigma$, whose solution is

$$
\sigma=-1+X+(6 \xi-1) \frac{\kappa(\alpha+\sqrt{2} e)}{c}
$$

where

$$
X \equiv \sqrt{\frac{\{c-(6 \xi-1) \kappa(\alpha+\sqrt{2} e)\}^{2}+(\alpha+\sqrt{2} e)^{2}\left\{6+(6 \xi-1) \kappa^{2}\right\}}{c^{2}}}
$$

is a quantity close to one. We have chosen the solution for $\sigma$ to approach zero in the $\epsilon, \tilde{\epsilon}, \alpha,(\xi-1 / 6) \rightarrow 0$ limit. The time derivative of the Hubble parameter is given by (6) and (7)

$$
\frac{\dot{H}}{H^{2}}=-\frac{2 \sigma+12 \xi \tilde{\epsilon}+(6 \xi-1)\left\{-\sigma+6 \frac{H \phi \pi}{V}+3(4 \xi-1) \frac{H^{2} \phi^{2}}{V}\right\}}{2+\sigma+(6 \xi-1)\left\{2 \frac{H \phi \pi}{V}+(6 \xi-1) \frac{H^{2} \phi^{2}}{V}\right\}} .
$$

Using the results above, (27) can be evaluated in terms of $e, \tilde{\epsilon}, \kappa, \xi$ and $\alpha$,

$$
=\frac{2 c(1-X-\tilde{\epsilon})+(6 \xi-1)\left[3 \kappa(\alpha+\sqrt{2} e)\left\{1+\xi\left(2+(6 \xi-1) \kappa^{2}\right)\right\}-c\left\{1-X+2 \tilde{\epsilon}-\kappa^{2}+\kappa^{2} \xi(3+X+2 \tilde{\epsilon})\right\}\right]}{\{c(1+X)-(6 \xi-1) \kappa(\alpha+\sqrt{2} e)\}\left\{1+\xi(6 \xi-1) \kappa^{2}\right\}} .
$$

Here it should be noted that $\alpha$ shows up either in the quadratic form $\alpha^{2}$, or with a prefactor $e \alpha$, $(6 \xi-1) \alpha$. The second order derivative is given by

$$
\begin{aligned}
& \frac{1}{H}\left(\frac{\dot{H}}{H^{2}}\right)=-\frac{6}{2+\sigma+(6 \xi-1)\left\{2 \frac{H \phi \pi}{V}+(6 \xi-1) \frac{H^{2} \phi^{2}}{V}\right\}}\left[(10 \xi-3) \sigma-2(7 \xi-1) \tilde{\epsilon}+(3 \xi-1) \frac{\pi V^{\prime}}{H V}+\xi \eta\left(\frac{\phi \pi}{M_{p}^{2} H}-\kappa^{2}\right)\right. \\
+ & \left.(6 \xi-1)\left\{2(4 \xi-3) \frac{H \phi \pi}{V}-(16 \xi-3) \frac{H^{2} \phi^{2}}{V}\right\}+\frac{\dot{H}}{H^{2}}\left\{(2 \xi-1) \sigma-4 \xi \tilde{\epsilon}+(6 \xi-1)\left\{2(2 \xi-1) \frac{H \phi \pi}{V}-(8 \xi-1) \frac{H^{2} \phi^{2}}{V}\right\}\right\}\right] .
\end{aligned}
$$

To avoid clutter, we do not lay out the explicit form of 
through $\alpha^{2}$, e $\alpha$, and $(6 \xi-1) \alpha$.

Now we turn to evaluating $\alpha$ itself. Though it is difficult to obtain the explicit value of $\alpha$, we can still derive its evolution equation. By differentiating both sides of (21) with respect to time and then substituting (7), one obtains

$$
\begin{gathered}
\frac{\dot{\alpha}}{H}=\alpha\left\{-2+2 \tilde{\epsilon}+\frac{\dot{H}}{H^{2}}+(\eta-2 \epsilon) \frac{V}{c M_{p}^{2} H^{2}}\right\} \\
+\sqrt{2} e\left\{c \sigma+\frac{\dot{H}}{H^{2}}+\frac{3}{c}\left(\eta_{c}-2 \epsilon+\eta \frac{V-3 M_{p}^{2} H^{2}}{3 M_{p}^{2} H^{2}}\right)\right\} \\
+2 \kappa\left(\xi-\frac{1}{6}\right)\left(2+\frac{\dot{H}}{H^{2}}\right) \frac{3 M_{p}^{2} H^{2}}{V}
\end{gathered}
$$

where we have made use of

$$
\eta \kappa=\sqrt{2} e\left\{\frac{3}{c}\left(\eta_{c}-\eta\right)+2-c\right\}
$$

along the way. The results above allow us to evaluate the r.h.s. of (30) in terms of $e, \epsilon, \tilde{\epsilon}, \eta_{c}, \eta, \kappa, \xi$ and $\alpha$. First let us consider the limit where exact conformal coupling is obtained and the three flatness parameters are negligible. Then the evolution equation (30) turns into

$$
\begin{aligned}
\frac{\dot{\alpha}}{H} & =\alpha \frac{6 c \eta-4 \sqrt{c^{4}+6 c^{2} \alpha^{2}}}{c^{2}+\sqrt{c^{4}+6 c^{2} \alpha^{2}}} \\
& \sim \alpha\left(-2+\frac{3 \eta}{c}\right)
\end{aligned}
$$

where the last line is an approximation valid in the case where $|\alpha| \ll|c|$. This shows that when $-2+3 \eta / c<0$, $\alpha$ damps as the universe expands during the inflationary era. When $\alpha$ becomes as small as the terms we have neglected in obtaining (32), the value of $\alpha$ settles down to that corresponding to the neglected source terms. Expanding the r.h.s. of (30) in terms of the flatness parameters and $(\xi-1 / 6)$, one can show

$$
\begin{aligned}
& \frac{\dot{\alpha}}{H}=\mathcal{O}\left(\epsilon, \epsilon^{1 / 2} \eta_{c}\right)+\mathcal{O}\left(\xi-\frac{1}{6}\right) \\
& \quad+\alpha\left\{-2+\frac{3 \eta}{c}+\mathcal{O}\left(\epsilon^{1 / 2}\right)+\mathcal{O}\left(\xi-\frac{1}{6}\right)\right\}+\mathcal{O}\left(\alpha^{2}\right) .
\end{aligned}
$$

Here, contributions from a nonexact conformal coupling are denoted by $\mathcal{O}\left((\xi-1 / 6)^{n}\right)$, which refers to products of $\mathcal{O}(1)$ factors and $(\xi-1 / 6)^{m}$ with $m \geq n$. It should be noted that we have not imposed any assumptions on the values of $\kappa$ and $\eta$. (34) shows that during inflation the value of $\alpha$ becomes of the order

$$
\alpha=\mathcal{O}\left(\epsilon, \epsilon^{1 / 2} \eta_{c}\right)+\mathcal{O}\left(\xi-\frac{1}{6}\right) .
$$

Now that we know the value of $\alpha$, we can compute the spectral index (19) and its running (20) in the modulated reheating scenario. Recalling how $\alpha$ showed up in time derivatives of the Hubble parameter, the leading order behavior of the tilt can be described without $\alpha$. We obtain

$$
\begin{aligned}
& n_{s}-1=-2 \tilde{\epsilon}-\left(\frac{12}{c^{2}}+\kappa^{2}\right) \epsilon+\mathcal{O}\left(\epsilon^{3 / 2}, \epsilon \eta_{c}\right)+\left(\xi-\frac{1}{6}\right)\left\{2 \kappa^{2}+\mathcal{O}\left(\epsilon^{1 / 2}\right)\right\}+\mathcal{O}\left(\left(\xi-\frac{1}{6}\right)^{2}\right) \\
& \begin{aligned}
\frac{d n_{s}}{d \ln k}=2\left(3-c+\frac{3}{c} \eta_{c}\right) \tilde{\epsilon}+\left(\frac{6(8-3 c)}{c^{2}}+(7-3 c) \kappa^{2}\right) \epsilon+\mathcal{O}\left(\epsilon^{3 / 2}, \epsilon \eta_{c}\right) \\
+\left(\xi-\frac{1}{6}\right)\left\{-4 \kappa^{2}+\mathcal{O}\left(\epsilon^{1 / 2}\right)\right\}+\mathcal{O}\left(\left(\xi-\frac{1}{6}\right)^{2}\right)
\end{aligned}
\end{aligned}
$$

We immediately see that $\left|d n_{s} / d \ln k\right|$ can become large, comparable to $\left|n_{s}-1\right|$. This is due to the fact that the derivatives of the flatness parameters do not necessarily become smaller than the parameters themselves. Since this is a generic feature of conformal inflation, a large running is expected even if we consider mechanisms other than modulated reheating for generating fluctuations (e.g. curvaton models [12, 13, 14]). Also, our results indicate that $(\xi-1 / 6)$-corrections can dominantly determine the values of the cosmological observables un- less $|\xi-1 / 6|$ is smaller than the flatness parameters.

Before ending this section, we should remark that when the inflaton is extremely close to its origin (i.e. $\left.\kappa^{2} \lesssim \mathcal{O}\left(\epsilon^{1 / 2}\right)\right)$, one may need to solve the evolution equation (30) of $\alpha$ in order to compute the $(\xi-1 / 6)$-corrections to the observables, since the "subleading" terms denoted by $(\xi-1 / 6) \mathcal{O}\left(\epsilon^{1 / 2}\right)$ in (36) and (37) become important. For example, (31) shows that such situations are realized when $|\eta| \gtrsim 1$ or when $c \approx 2$. See also Appendix A2 for arguments on the validity of our results before the 
inflaton trajectory approaches the attractor.

\section{APPLICATION TO WARPED BRANE INFLATION}

Let us now apply the results obtained in the previous section to a specific model. Here we consider the warped brane inflation model [5, 6, 7, 8, 9, 10], where the universe experiences inflation while a D3-brane moves towards the tip of a flux compactified warped throat. The D3-brane is pulled by a stack of $\overline{\mathrm{D} 3}$-branes sitting at the tip. If the position of the D3-brane is a conformally coupled scalar (for a discussion on this issue, see e.g. [15]), then this model serves as a realization of conformal inflation. The attractor behavior in this case is demonstrated in [1] by the phase portrait method.

Considering a throat whose geometry is $A d S_{5} \times X_{5}$, the potential of the inflaton takes the form

$$
V(\phi)=2 p h_{0}^{4} T_{3}\left(1-\frac{h_{0}^{4} T_{3}^{2} R^{4}}{N \phi^{4}}\right)
$$

Here, the inflaton is related to the radial position $\rho$ of the D3 through $\phi=\sqrt{T_{3}} \rho, p$ is the number of $\overline{\mathrm{D} 3}$ s at the tip, $h_{0}=\rho_{0} / R$ is the warping at the tip, $T_{3}=1 /(2 \pi)^{3} g_{s}\left(\alpha^{\prime}\right)^{2}$ is the D3 tension, $R^{4}=2^{2} \pi^{4} g_{s}\left(\alpha^{\prime}\right)^{2} N / \operatorname{Vol}\left(X_{5}\right)$ is the AdS radius of the throat, $\operatorname{Vol}\left(X_{5}\right)$ is the dimensionless volume of the base space $X_{5}$, and $N(>1)$ is the 5 -form charge.

The conformally coupled inflaton satisfies $\dot{\phi} \approx-\phi H$ during inflation, hence the number of $e$-foldings generated is ${ }^{4}$

$$
\mathcal{N} \approx \log \left(\frac{\rho_{i}}{\rho_{f}}\right) \approx-\log \left(\frac{h_{0}}{\lambda_{i}}\right)
$$

where we have set the initial position of the D3 by a dimensionless constant $\lambda_{i}$ as $\rho_{i}=\lambda_{i} R$, and assumed inflation to end when the D3 approaches the tip $\rho_{f} \approx \rho_{0}$. This shows that in order to obtain enough $e$-foldings, the throat should be strongly warped $h_{0} / \lambda_{i} \ll 1$.

Assuming $\alpha$ in (21) to be sufficiently damped when the perturbations of the $\mathrm{CMB}$ scale are generated, the scale dependence of the perturbations can be computed by (36) and (37). We parametrize the position of the D3 when the $\mathrm{CMB}$ scale was originally produced by $\rho_{\mathrm{CMB}}=\lambda R$. Furthermore, for simplicity, we ignore the compactified bulk to which the throat is glued, hence

$$
M_{p}^{2} \simeq \frac{2}{(2 \pi)^{7} g_{s}^{2}\left(\alpha^{\prime}\right)^{4}} \int_{\rho_{0}}^{R} d \rho \operatorname{Vol}\left(X_{5}\right) \frac{\rho^{5}}{h^{4}} \simeq \frac{\operatorname{Vol}\left(X_{5}\right) R^{6}}{(2 \pi)^{7} g_{s}^{2}\left(\alpha^{\prime}\right)^{4}}
$$

\footnotetext{
4 The deviation from an exact conformal coupling will appear as an $\mathcal{O}(\xi-1 / 6)$ correction to this result, which we can ignore.
}

Then under the assumption $h_{0} / \lambda \ll 1$, the flatness parameters and $\kappa$ can be calculated (note that $c=7$ ),

$$
\epsilon \simeq \frac{2 h_{0}^{8}}{\lambda^{10} N}, \tilde{\epsilon} \simeq \frac{2 h_{0}^{4}}{\lambda^{4} N}, \eta_{c}=\eta \simeq-\frac{5 h_{0}^{4}}{\lambda^{6}}, \kappa^{2} \simeq \frac{4 \lambda^{2}}{N} .
$$

Since $\epsilon$ is extremely small compared to the other flatness parameters, the cosmological observables can be estimated as follows:

$$
\begin{aligned}
n_{s}-1 & \simeq-2 \tilde{\epsilon}+2 \kappa^{2}\left(\xi-\frac{1}{6}\right) \\
& \simeq-\frac{4}{N}\left\{\frac{h_{0}^{4}}{\lambda^{4}}-2 \lambda^{2}\left(\xi-\frac{1}{6}\right)\right\} \\
\frac{d n_{s}}{d \ln k} & \simeq-8 \tilde{\epsilon}-4 \kappa^{2}\left(\xi-\frac{1}{6}\right) \\
& \simeq-\frac{16}{N}\left\{\frac{h_{0}^{4}}{\lambda^{4}}+\lambda^{2}\left(\xi-\frac{1}{6}\right)\right\} .
\end{aligned}
$$

The 5-year WMAP $+\mathrm{BAO}+\mathrm{SN}$ data give bounds $n_{s}=$ $1.022_{-0.042}^{+0.043}(68 \% \mathrm{CL})$ and $d n_{s} / d \ln k=-0.032_{-0.020}^{+0.021}$ (68\% CL) when tensor mode perturbations are negligible [11]. Since the $h_{0}^{4} / \lambda^{4}$ terms are too small to be constrained by the observational bounds, we ignore them. Then the observables are determined only by the inflaton's deviation from an exact conformal coupling,

$$
n_{s}-1 \simeq 8 \frac{\lambda^{2}}{N}\left(\xi-\frac{1}{6}\right), \frac{d n_{s}}{d \ln k} \simeq-16 \frac{\lambda^{2}}{N}\left(\xi-\frac{1}{6}\right) .
$$

It is easy to see that the spectral index and its running are related by

$$
\frac{d n_{s}}{d \ln k} \simeq-2\left(n_{s}-1\right)
$$

Using the observational bound on $d n_{s} / d \ln k$ to constrain $\lambda^{2}(\xi-1 / 6) / N$

$$
0.001 \lesssim \frac{\lambda^{2}}{N}\left(\xi-\frac{1}{6}\right) \lesssim 0.003
$$

If we further make use of this bound to constrain the spectral index, we obtain

$$
0.006 \lesssim n_{s}-1 \lesssim 0.026
$$

Thus the $1 \sigma$ observational bound on the running allows us to predict a blue tilt for the warped brane inflation model. Moreover, the values of the observables were dominantly determined by the inflaton's deviation from a conformal coupling. The bound (48) suggests that this deviation can be fairly large, e.g., $(\xi-1 / 6) \sim 10^{-1}$ when $\lambda \sim 1, N \sim 10^{2}$. However, note that if $N(\lambda)$ is larger (smaller), then such a large $(\xi-1 / 6)$ would indicate the breakdown of the procedure of expanding values in terms of $(\xi-1 / 6)$. In such case numerical analysis would be required. 


\section{CONCLUSION}

In this paper, we have investigated the scale dependence of the density perturbations that are generated through modulated reheating after conformal inflation. We have written down the spectral index and its running in terms of the flatness parameters and the inflaton's deviation from an exact conformal coupling. The result indicates the presence of a large running, which reflects the fact that the derivatives of the flatness parameters are not necessarily small. This is a basic feature of conformal inflation, and it is expected that a large running will be obtained even if we consider other mechanisms for generating fluctuations (e.g. curvaton models [12, 13, 14]). It would be interesting to analyze systematically the differences in perturbations generated through conformal inflation models and standard minimal ones.

We also applied our results to the warped brane inflation model, where it was shown that observables were dominantly determined by the deviation from the conformal coupling. We have shown that the spectral index and its running are related by (47) for this model. Since the running is highly constrained by the WMAP5 data, a stringent bound on the coupling of the inflaton to gravity was obtained. Also, comparison with the WMAP5 data suggested a blue tilt of the spectrum. (However, we note that constraints on the spectrum can be altered if cosmic strings are produced after brane inflation, see [16, 17, 18] and references therein.)

While our analysis focused on general aspects of modulated reheating after conformal inflation, in this paper we have not presented the light modulus responsible for generating fluctuations in a concrete setup. In the warped brane inflation case, potential candidates for such light fields are angular positions of the D3( $\overline{\mathrm{D} 3})$-branes sitting in throats with angular isometries, and/or axions associated with shift symmetries of the Kähler potential. They may have negligible effects on the inflaton dynamics during inflation, but one can expect such fields to play the role of the moduli fields and eventually generate density perturbations. For further study, it is important to come up with an explicit realization of our mechanism based on fundamental theories. We leave this for future work.

We have focused on the tilt of the density perturbations, but it would also be worthwhile to study nonGaussianities in conformal inflation models. One of the general lessons of our work is that a nonminimal coupling with gravity can drastically change the behavior of inflation. A special feature of conformal inflation is that higher-order time derivatives of the Hubble parameter have large values. Cosmological observations are imposing (not necessarily direct but) important constraints on such features even at the present stage.

\section{Acknowledgments}

We are grateful to Issha Kayo, Shunichiro Kinoshita, Lev Kofman, and Tadashi Takayanagi for useful discussions. T.K. would also like to thank Katsuhiko Sato for his continuous support. The work of S.M. was supported in part by MEXT through a Grant-in-Aid for Young Scientists (B) No. 17740134, and by JSPS through a Grantin-Aid for Creative Scientific Research No. 19GS0219 and through a Grant-in-Aid for Scientific Research (B) No. 19340054. This work was supported by World Premier International Research Center Initiative (WPI Initiative), MEXT, Japan. This work was also supported in part by the Global COE Program "the Physical Sciences Frontier," MEXT, Japan.

\section{APPENDIX A: DETAILED DISCUSSIONS OF CONFORMAL INFLATION}

We give detailed arguments on conformal inflation. Throughout this appendix, we consider the case of exact conformal coupling $\xi=1 / 6$.

\section{Selection Criterion for $c$}

Let us introduce a dimensionless parameter $\beta$

$$
c H \pi=-V^{\prime}(1+\beta)
$$

which measures the validity of the approximation (9) during conformal inflation. (One may wonder why we have used $\alpha$ as defined in (21) in the body of this paper. This is due to the sharp breakdown of the approximation (9) when there is a deviation from $\xi=1 / 6$, in which case $\beta$ can become significantly large.) Then by following the same procedure as for $\alpha$, we can derive the evolution equation of $\beta$,

$$
\frac{\dot{\beta}}{H}=\mathcal{O}\left(\epsilon^{1 / 2}, \eta_{c}\right)-\beta\left\{c-\frac{3 \eta}{c}+\mathcal{O}\left(\epsilon^{1 / 2}, \eta_{c}\right)\right\}+\mathcal{O}\left(\beta^{2}\right)
$$

This shows that the condition

$$
c-\frac{3 \eta}{c}>0
$$

is required for $\beta$ to decay and for (9) to be an inflationary attractor. A2 also indicates that conformal inflation is unstable for small $|c|$.

When $\eta$ is negligible, the inflationary condition $\eta_{c} / c^{2} \approx$ 0 gives

$$
c=2-\zeta
$$

where we have defined $\zeta \equiv \phi V^{\prime \prime} / V^{\prime}$. Then the condition (A3) demands

$$
\zeta<2
$$


in order for the existence of the attractor.

On the other hand, when $\eta$ is non-negligible, $\eta_{c} / c^{2} \approx 0$ sets the constant $c$ to either of

$$
c_{ \pm}=\frac{1}{2}\left\{2-\zeta \pm \sqrt{(2-\zeta)^{2}-12 \eta}\right\}
$$

Considering the condition (A3), one can easily show

$$
c_{ \pm}-\frac{3 \eta}{c_{ \pm}}= \pm \sqrt{(2-\zeta)^{2}-12 \eta}
$$

Thus we conclude that (9) with the larger solution $c_{+}$is the attractor of conformal inflation.

\section{2. (Non)Relation Between the Inflationary Attractor and Predictions of Cosmological Observables}

We have seen in the previous subsection that once $|\beta|$ becomes small, (9) continues to hold as long as the condition (A3) is satisfied. However, in actual cases, the nonlinear terms in (A2) may initially prevent $|\beta|$ from dropping down to a value smaller than unity, and it may take some time for the inflaton to fall into the attractor. Here, we point out that whether or not the inflaton trajectory approaches the attractor (9) is not directly related to the validness of our results (36) and (37) on cosmological observables.

This can easily be understood by recalling how $\alpha$ (or $\beta$ ) showed up in (28). Taking into account $\alpha=\sqrt{2} e \beta$, one can obtain the full expression for the spectral index in the exactly conformally coupled case

$$
\begin{aligned}
n_{s}-1 & =\frac{4(1-\tilde{\epsilon})-4 \sqrt{1+\frac{12}{c^{2}}(1+\beta)^{2} \epsilon}}{3-2 \tilde{\epsilon}-\sqrt{1+\frac{12}{c^{2}}(1+\beta)^{2} \epsilon}} \\
& =-2 \tilde{\epsilon}-\left(\frac{12}{c^{2}}(1+\beta)^{2}+\kappa^{2}\right) \epsilon+\mathcal{O}\left(\epsilon^{3 / 2}\right),
\end{aligned}
$$

where in the last line we have performed the flatness parameter expansion without imposing any assumptions on the value of $\beta$. This implies that even if the inflaton trajectory has not settled down to the attractor (9) (i.e. $|\beta| \gtrsim 1$ ), if $\epsilon$ is small enough to satisfy $|\tilde{\epsilon}| \gg \beta^{2} \epsilon$ (i.e. $\left.\phi^{2} / \widetilde{M}_{p}^{2} \gg \beta^{4} \epsilon\right)$, then the $\epsilon$ term containing $\beta$ is negligible and our result (36) is valid. Similar arguments can also be made for the running.
[1] L. Kofman and S. Mukohyama, Phys. Rev. D 77, 043519 (2008) arXiv:0709.1952 [hep-th]].

[2] N. Makino and M. Sasaki, Prog. Theor. Phys. 86, 103 (1991).

[3] G. Dvali, A. Gruzinov and M. Zaldarriaga, Phys. Rev. D 69, 023505 (2004) arXiv:astro-ph/0303591.

[4] L. Kofman, arXiv:astro-ph/0303614.

[5] S. Kachru, R. Kallosh, A. Linde, J. M. Maldacena, L. P. McAllister and S. P. Trivedi, JCAP 0310, 013 (2003) arXiv:hep-th/0308055.

[6] D. Baumann, A. Dymarsky, I. R. Klebanov, J. M. Maldacena, L. P. McAllister and A. Murugan, JHEP 0611, 031 (2006) arXiv:hep-th/0607050.

[7] D. Baumann, A. Dymarsky, I. R. Klebanov, L. McAllister and P. J. Steinhardt, Phys. Rev. Lett. 99, 141601 (2007) arXiv:0705.3837 [hep-th]].

[8] A. Krause and E. Pajer, JCAP 0807, 023 (2008) arXiv:0705.4682 [hep-th]].

[9] D. Baumann, A. Dymarsky, I. R. Klebanov and L. McAllister, JCAP 0801, 024 (2008) arXiv:0706.0360 [hep-th]].
[10] D. Baumann, A. Dymarsky, S. Kachru, I. R. Klebanov and L. McAllister, arXiv:0808.2811 [hep-th].

[11] E. Komatsu et al. [WMAP Collaboration], Astrophys. J. Suppl. 180, 330 (2009) arXiv:0803.0547 [astro-ph]].

[12] K. Enqvist and M. S. Sloth, Nucl. Phys. B 626, 395 (2002) arXiv:hep-ph/0109214.

[13] D. H. Lyth and D. Wands, Phys. Lett. B 524, 5 (2002) arXiv:hep-ph/0110002.

[14] T. Moroi and T. Takahashi, Phys. Lett. B 522, 215 (2001) [Erratum-ibid. B 539, 303 (2002)] arXiv:hep-ph/0110096.

[15] N. Seiberg and E. Witten, JHEP 9904, 017 (1999) arXiv:hep-th/9903224.

[16] J. Polchinski, arXiv:hep-th/0412244

[17] N. Bevis, M. Hindmarsh, M. Kunz and J. Urrestilla, Phys. Rev. Lett. 100, 021301 (2008) arXiv:astro-ph/0702223.

[18] R. A. Battye, B. Garbrecht, A. Moss and H. Stoica, JCAP 0801, 020 (2008) arXiv:0710.1541 [astro-ph]]. 